\title{
Correction to: Essentials in saline pharmacology for nasal or respiratory hygiene in times of COVID-19
}

\author{
Suzy Huijghebaert ${ }^{1}$ (D) $\cdot$ Levi Hoste $^{2,3}$ (1) $\cdot$ Guido Vanham $^{4}$
}

Published online: 24 April 2021

C Springer-Verlag GmbH Germany, part of Springer Nature 2021

Correction to: European Journal of Clinical Pharmacology
https://doi.org/10.1007/s00228-021-03102-3

The correct presentation of the Author names are shown in this paper.

The Original article has been corrected.

Suzy Huijghebaert

s.huijghebaert@scarlet.be; s.huijghebaert@gmail.com;

OralMedDevs@gmail.com

1 Independent Research Support, La Hulpe, Belgium

2 Pediatric Pulmonology, Infectious Diseases and Immunology,

Ghent University Hospital, Ghent, Belgium

3 Primary Immunodeficiency Research Lab, Center for Primary Immunodeficiency Ghent, Jeffrey Modell Diagnosis and Research Center, Ghent University Hospital, Ghent, Belgium

4 Department of Biomedical Sciences, Institute of Tropical Medicine and University of Antwerp, Antwerp, Belgium 